\title{
Reduction in proteinuria after immunosuppres- sive therapy and long-term kidney outcomes in patients with immunoglobulin A nephropathy
}

Shin Chan Kang ${ }^{1}$, Hyung Woo Kim ${ }^{1}$, Tae Ik Chang ${ }^{2}$, Ea Wha Kang ${ }^{2}$, Beom Jin Lim³ Jung Tak Park ${ }^{1}$, Tae-Hyun Yoo $^{1}$, Hyeon Joo Jeong ${ }^{3}$, Shin-Wook Kang ${ }^{1,4}$, and Seung Hyeok Han ${ }^{1}$

${ }^{1}$ Department of Internal Medicine, Institute of Kidney Disease, Yonsei University College of Medicine, Seoul; ${ }^{2}$ Division of Nephrology, Department of Internal Medicine, National Health Insurance Service Ilsan Hospital, Goyang; ${ }^{3}$ Department of Pathology, Yonsei University College of Medicine, Seoul; ${ }^{4}$ Severance Biomedical Science Institute, Brain Korea 21 PLUS, Yonsei University, Seoul, Korea
Received: May 19, 2020

Revised : June 22, 2020

Accepted: June 25, 2020

\section{Correspondence to}

Seung Hyeok Han, M.D.

Division of Nephrology,

Department of Internal

Medicine, Yonsei University

College of Medicine, 50-1

Yonsei-ro, Seodaemun-gu, Seoul 03722, Korea

Tel: +82-2-2228-1984

Fax: +82-2-393-6884

E-mail:hansh@yuhs.ac

https://orcid.org/o0oo-ooo1-

7923-5635
Background/Aims: Despite controversy regarding the benefits of immunosuppressive therapy in immunoglobulin A nephropathy (IgAN), clinical outcomes may vary depending on the patient's responsiveness to this therapy. This study evaluated long-term kidney outcomes according to the extent of proteinuria reduction after immunosuppression in IgAN patients.

Methods: Among 927 patients with biopsy-proven IgAN, 127 patients underwent immunosuppression. Time-averaged urine protein-creatinine ratio before and within 1 year after start of immunosuppression were calculated, and responsiveness to immunosuppression was assessed as the reduction of proteinuria between the two periods. Patients were classified into tertiles according to the extent of proteinuria reduction. We compared the slopes of estimated glomerular filtration rate (eGFR) decline using a linear mixed model, and estimated hazard ratios (HRs) for disease progression (defined as development of $\mathrm{a} \geq 30 \%$ decline in eGFR or end-stage renal disease) using a Cox proportional hazard model.

Results: Median extent of proteinuria reduction was $-2.1,-0.9$, and $-0.2 \mathrm{~g} / \mathrm{gCr}$ in the first, second, and third tertiles, respectively. There were concomitant changes in the slopes of annual eGFR decline: $-2.03,-2.44$, and $-4.62 \mathrm{~mL} / \mathrm{min} / 1.73 \mathrm{~m}^{2}$ among the first, second, and third tertiles, respectively. In multivariable Cox analysis, the HRs ( $95 \%$ confidence intervals) for disease progression were 0.30 ( 0.12 to 0.74 ) in the first tertile and 0.70 ( 0.34 to 1.45 ) in the second tertile compared with the thirdtertile.

Conclusions: This study showed that greater proteinuria reduction after immunosuppression was associated with a lower risk of disease progression in patients with IgAN, suggesting that responsiveness to immunosuppression may be an important determinant of kidney outcomes.

Keywords: Immunoglobulin A nephropathy; Immunosuppression; Proteinuria

\section{INTRODUCTION}

Immunoglobulin A nephropathy (IgAN) is the most common primary glomerulonephritis worldwide, and the prevalence of IgAN is higher in East Asians than in other ethnicities $[1,2]$. It is not a totally benign disease, 
because $20 \%$ to $40 \%$ of patients progress to end-stage renal disease (ESRD) within 20 years after diagnosis [3,4]. Therefore, timely and proper treatment is required to delay the progression of IgAN.

Multiple factors are involved in the development of IgAN, but a key step in pathogenesis is the formation of immune complexes by binding of autoantibodies to galactose-deficient IgA1 [5]. This immunologic mechanism has provided the rationale for immunosuppressive therapy in the management of IgAN. In fact, several randomized controlled trials have published findings suggesting that corticosteroid treatment reduces proteinuria and improves kidney survival in patients with IgAN [6-8]. Based on these results, the Kidney Disease: Improving Global Outcome (KDIGO) clinical guidelines published in 2012 included indications for corticosteroid use as a therapeutic option in IgAN. However, evidence level for this suggestion is low, and the first line therapy is still supportive care involving control of blood pressure and proteinuria with renin-angiotensin-aldosterone system (RAAS) blockers. Immunosuppressive therapy can be considered in patients with persistently elevated urinary protein excretion ( $\geq 1 \mathrm{~g}$ /day) after 3 to 6 months of maximal supportive care [9]. Nevertheless, the use of immunosuppression is not widely favored because not all patients show consistent responsiveness to corticosteroids and there are many concerns regarding the serious adverse effects of immunosuppression [10]. Moreover, a recent German study failed to demonstrate superiority of immunosuppressive treatment over intensive supportive care in IgAN patients with persistent proteinuria [11]. For this reason, the panels from the KDIGO controversies conference in 2018 do not strongly support use of immunosuppression in patients with IgAN [12].

Despite such controversy, immunosuppression is still a therapeutic option in real-world clinical practice and clinical outcomes may vary depending on patients' responsiveness to this therapy. Several studies have investigated factors associated with responsiveness to immunosuppressive therapy and shown that immunosuppressive drugs can be effective, particularly in the presence of existence of endocapillary proliferation, preserved renal function, and a higher amount of proteinuria $[13,14]$. Among these, proteinuria has long been recognized as the strongest risk factor for the progression of IgAN [15-18]. There are various patterns of proteinuria response to immunosuppression. Some patients show sustained remission, while others experience a rebound increase in proteinuria after discontinuation of immunosuppressive drugs. However, few studies have examined the relationship between the degree of proteinuria reduction after immunosuppressive therapy and longterm kidney outcomes. Therefore, in this retrospective, observational study, we used proteinuria reduction as a surrogate marker for responsiveness to immunosuppressive therapy and investigated the progression of kidney disease in patients with IgAN treated with immunosuppressive therapy.

\section{METHODS}

\section{Patient selection}

A flow diagram of the selection of patients is presented in Fig. 1. Among 927 patients with biopsy-proven IgAN at Yonsei University Severance Hospital and National Health Insurance Services Ilsan Hospital between November 2005 and November 2017, a total of 174 patients were considered eligible for the study after excluding those with secondary conditions associated with IgAN $(\mathrm{n}=38)$ and those not treated with immunosuppression $(\mathrm{n}=715)$. Among the patients, those with the following conditions were excluded: age at biopsy $<18$ years $(n=2)$; started dialysis before biopsy $(\mathrm{n}=13)$; baseline estimated glomerular filtration rate $(\mathrm{eGFR})<30 \mathrm{~mL} / \mathrm{min} / 1.73 \mathrm{~m}^{2}$ $(\mathrm{n}=8)$; started immunosuppression before biopsy ( $\mathrm{n}=$ 8); immunosuppression duration < 30 days $(n=5)$. We further excluded patients with less than two visits after biopsy, those with a follow-up duration of less than 3 months $(n=6)$, and those with less than two eGFR and proteinuria measurements either before or after the start of immunosuppressive therapy $(\mathrm{n}=5)$. Two patients with a $\mathrm{T} 2$ lesion were excluded since they also had eGFR less than $30 \mathrm{~mL} / \mathrm{min} / 1.73 \mathrm{~m}^{2}$ at baseline. Therefore, 127 patients were included in the final analysis. Patients were classified into tertiles according to the extent of time-averaged proteinuria (TAP) reduction between before and within 1 year from the start of immunosuppression (Ter1, first tertile; Ter2, second tertile; Ter3, third tertile).

The study was conducted in accordance with the Dec- 


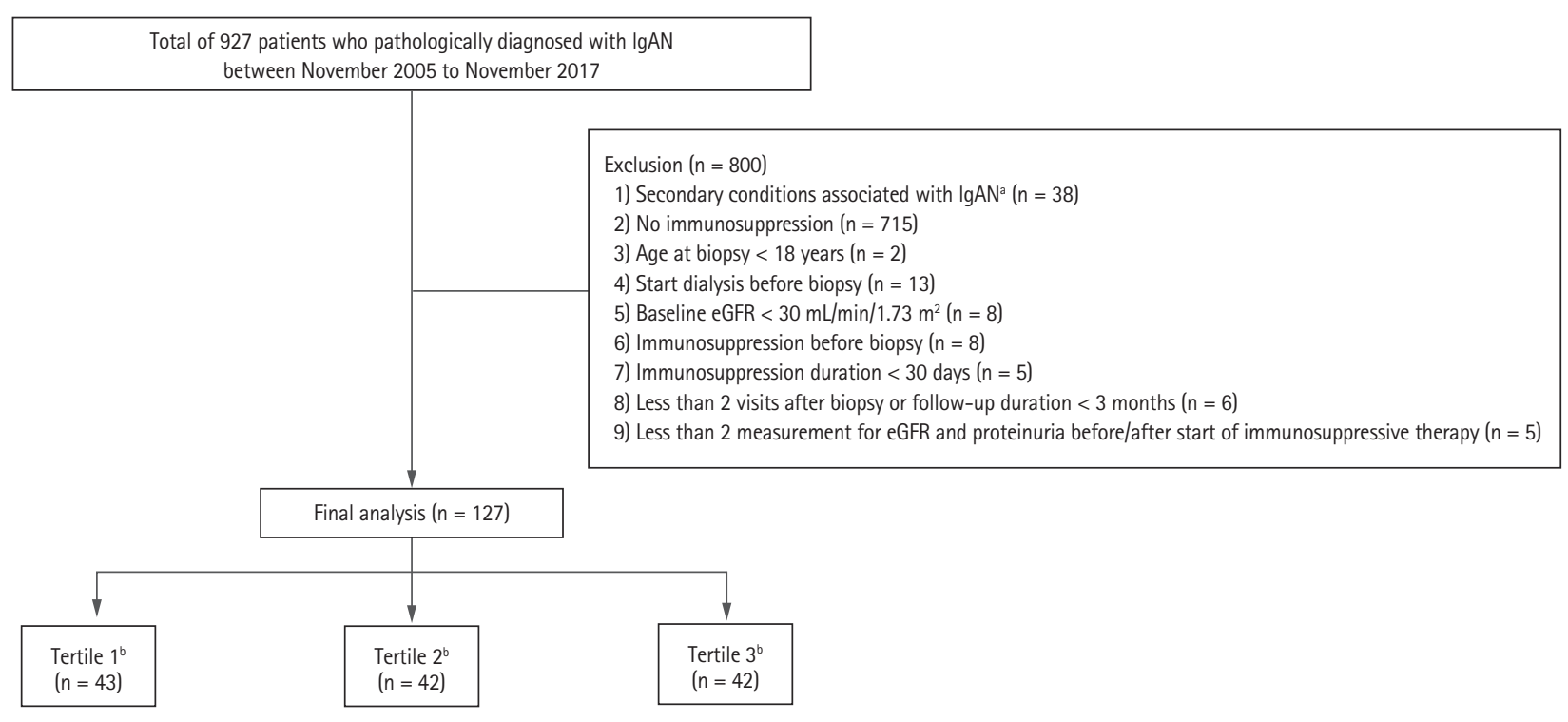

Figure 1. Flow diagram of the study. IgAN, immunoglobulin A nephropathy; eGFR, estimated glomerular filtration rate. ${ }^{a}$ Secondary conditions included infection-associated glomerulonephritis and combined autoimmune disease such as Henoch-Schonlein purpura, systemic lupus nephritis, Sjogren's syndrome and rheumatoid arthritis, ${ }^{\text {b}}$ Patients were categorized into tertiles according to the extent of time-averaged proteinuria reduction after immunosuppression.

laration of Helsinki and approved by the Institutional Review Board of Yonsei University Health System Clinical Trial Center (IRB no. 4-2019-0548). Because the study was retrospective and patients were de-identified, the need for written consent from the patients was waived.

\section{Data collection}

Baseline demographic and clinical data including age, sex, anthropometric measurements, comorbidities, medication history, and blood pressure were collected at the time of biopsy and at the start of immunosuppressive therapy. Laboratory parameters such as blood urea nitrogen, serum creatinine, hemoglobin, total protein, serum albumin, high-density lipoprotein cholesterol (HDL-C), low-density lipoprotein cholesterol (LDL-C), triglycerides, serum electrolytes, spot urine protein, and urine creatinine were measured. Serum creatinine concentration was measured by the isotope dilution mass spectroscopy traceable kinetic Jaffe assay. The eGFR was calculated using the Chronic Kidney Disease Epidemiology Collaboration (CKD-EPI) equation [19]. During the follow-up period, proteinuria was assessed using the spot urine protein-to-creatinine ratio (UPCR) because 24 hour urine protein quantification was not feasible at every visit. Pathologic findings were presented using the Oxford classification criteria [13,20]. All renal biopsy specimens were reassessed by one pathologist blinded to the patients' clinical data. Because this was not a randomized controlled study, there was no pre-set regimen, dosage, or duration of immunosuppression. However, we generally followed the glucocorticoid protocol suggested by Pozzi et al. [6] or Manno et al. [7] during the initial 6 months. Additional immunosuppression with other drugs was performed at the physician's discretion and based on disease status. In this study, we used TAP, because proteinuria at a single time-point cannot reflect overall proteinuria status. TAP was calculated as the weighted mean of UPCR measurements, with weights representing time elapsed since the previous measurement [10]. Pre- and post-immunosuppression (IS) TAPs were determined using all UPCR measurements within 12 months before and after the start of immunosuppression, respectively. Responsiveness to immunosuppression was represented as the reduction in TAP.

\section{Study outcomes}

We hypothesized that kidney function decline would be slower over time in patients with greater proteinuria re- 
duction. Our primary outcome of interest was the slope of eGFR decline after the start of immunosuppression. Secondary outcome was disease progression, defined as development of $\mathrm{a} \geq 30 \%$ decline in eGFR or incident ESRD [21]. To avoid the possibility of transient eGFR reduction being classified as disease progression, at least two consecutive measurements of $\mathrm{a} \geq 30 \%$ decline in eGFR were ascertained. The first of these consecutive measurements was retrospectively designated as the study endpoint. ESRD was defined as initiation of dialysis or kidney transplantation.

\section{Statistical analysis}

Continuous variables are expressed as means and standard deviations and categorical variables as absolute numbers with percentages. Comparisons between groups were performed using Student's $t$ test or analysis of variance for continuous variables and the chisquare test or Fisher's exact test for categorical variables. The Shapiro-Wilk test was performed to determine the normality of the distribution of the parameters. If the resulting data did not show a normal distribution, medians and interquartile ranges were reported; the Mann-Whitney $U$ test or Kruskal-Wallis test was then used to compare groups. We computed the slope of eGFR decline in $\mathrm{mL} / \mathrm{min} / 1.73 \mathrm{~m}^{2}$ per year by applying a linear mixed-effect model and a sandwich estimator of variance to account for correlation within the same subject. Following terms were included in the linear mixed-effect model: age at start of immunosuppression, sex, time from biopsy to start of immunosuppression, baseline eGFR, pre-IS TAP, time-averaged SBP, serum albumin, and presence of endocapillary hypercellularity. We assumed that patients with proteinuria reduction would continue to show improvement in proteinuria after completion of immunosuppressive therapy. Most immunosuppressive therapy was completed within 12 months. Thus, we performed linear mixed-effect analysis with a turning point at 1 year after the start of immunosuppression. Cox proportional hazard model was constructed to determine if there was an independent association between TAP reduction and disease progression. The model was adjusted for multiple variables that showed statistical significance $(p<0.05)$ in univariate analysis or had clinical significance in previous studies. Model 1 was the crude model without adjustment.
Model 2 was adjusted for age at the start of immunosuppression, sex, time from biopsy to start of immunosuppressive therapy, and baseline eGFR. Model 3 additionally included pre-IS TAP and time-averaged SBP. Finally, Model 4 was created after further adjustment for serum albumin, the presence of endocapillary hypercellularity, and use of renin-angiotensin system blockers at start of immunosuppressive therapy. All results were expressed as hazard ratios (HRs) and 95\% confidence intervals (CIs). Adjusted cumulative renal survival curves were plotted after the same adjustments specified for the Cox models. Survival time was defined as the time interval between the time of starting immunosuppression and the onset of the endpoint or last follow-up, and follow-up loss was censored. Restricted cubic splines were used to determine the association between the degree of proteinuria reduction as a continuous variable and the $\mathrm{H}$ ) for disease progression. For all analyses, a $p$ value of less than 0.05 was considered statistically significant. All statistical analyses were performed using Stata version 15.1 for Windows (StataCorp LLC, College Station, TX, USA).

\section{RESULTS}

\section{Baseline characteristics}

Decrements in TAP in each tertile group are shown in Supplementary Fig. 1; Terı showed the greatest proteinuria reduction. Baseline characteristics of the patients at the time of kidney biopsy are described in Supplementary Table 1. Median proteinuria was $1.8 \mathrm{~g} / \mathrm{gCr}$ (range, 1.2 to 3.3 ), median eGFR was $79.3 \mathrm{~mL} / \mathrm{min} / 1.73 \mathrm{~m}^{2}$ (range, 61.2 to 103.3$)$, and mean age was $39.6 \pm 11.3$ years. Seventynine patients $(62.2 \%)$ were female and 104 (81.9\%) patients had a history of hypertension. Baseline proteinuria level was significantly higher in Terı than in the other two groups. In addition, patients in this group had a higher cholesterol level and lower albumin level than those in the other two groups. There were no significant differences in kidney function, blood pressure, kidney function, or proportion of Oxford-MEST-C components among tertile groups. At the start of immunosuppression, median proteinuria was $2.1 \mathrm{~g} / \mathrm{gCr}$ (range, 1.4 to 3.5), and median pre-IS and post-IS TAPs were $1.9 \mathrm{~g} / \mathrm{gCr}$ (range, 1.4 to 3.1) and 1.1 g/gCr (range, 0.6 to 1.6), respec- 
tively (Table 1). Median reduction in TAP was $-2.1 \mathrm{~g} / \mathrm{gCr}$ (range, -3.7 to -1.5 ), $-0.9 \mathrm{~g} / \mathrm{gCr}$ (range, -1.0 to -0.7 ), and $-0.2 \mathrm{~g} / \mathrm{gCr}$ (range, -0.4 to 0.5 ), in Ter1, Ter2, and Ter3, respectively. Baseline eGFR at the initiation of immunosuppression was significantly lower in Terz than in the other two groups. In addition, the time elapsed from biopsy to the initiation of immunosuppressive therapy was significantly longer in Ter3 than Ter1 and Ter2. Immunosuppression medications included glucocorticoids, cyclophosphamide, mycophenolate, and calcineurin inhibitors. All patients received corticosteroids as first line immunosuppression agents. There were no significant differences in the use and cumulative dose of immunosuppressive drugs or duration of treatment among tertiles.

\section{Changes in eGFR decline rates according to pro- teinuria reduction}

During a mean follow-up of 5.1 years, the overall eGFR decline slope of all patients was $-2.97 \mathrm{~mL} / \mathrm{min} / 1.73 \mathrm{~m}^{2} /$ year (range, -3.99 to -1.95 ) (Table 2). There were significant differences in kidney function decline rates across the three tertile groups with corresponding slopes of annual eGFR decline of $-2.03 \mathrm{~mL} / \mathrm{min} / 1.73 \mathrm{~m}^{2} /$ year (range, -2.95 to -1.11 ), $-2.44 \mathrm{~mL} / \mathrm{min} / 1.73 \mathrm{~m}^{2} /$ year (range, $-4.71,-0.17$ ), and $-4.62 \mathrm{~mL} / \mathrm{min} / 1.73 \mathrm{~m}^{2} /$ year (range, -6.52 , -2.72) for Ter1, Ter2, and Ter3, respectively. Predicted eGFR was derived from a piecewise linear mixed-effect model, and slower eGFR decline was noted in patients with higher TAP reduction; these differences in predicted eGFR persisted over time after completion of immunosuppressive therapy (Fig. 2).

\section{Proteinuria reduction and disease progression}

To compare risk of disease progression among tertile groups, a multivariable-adjusted Cox proportional hazard model was constructed. During the follow-up period, disease progression occurred in 14 (32.6\%), 14 (33.3\%), and 25 (59.5\%) patients in Ter1, Ter2, and Ter3, respectively. Corresponding incidence rates were 75.0, 91.8, and 143.1 per 1,000 person-years for the respective tertile groups. In multivariable Cox proportional hazard analysis, the largest reduction in proteinuria was associated with a lower risk of disease progression. HRs (95\% CI) for Terı and Ter2 compared to Ter3 were 0.33 (range, 0.14 to 0.79 ) and 0.75 (range, 0.36 to 1.54 ), respectively
(Table 3). Adjusted survival curves showed that time to development of disease progression was significantly longer in patients with greater TAP reduction than in those with the lowest TAP reduction (Fig. 3). Restricted cubic spline curve also showed a non-linear relationship between the extent of proteinuria reduction and the risk for kidney disease progression (Fig. 4). This association was more pronounced in patients with proteinuria $<3.0$ $\mathrm{g} / \mathrm{gCr}$, but it did not reach statistical significance (Supplementary Table 2).

\section{Changes in proteinuria after completion of immu- nosuppression}

Finally, we wondered if proteinuria levels may change after completion of immunosuppression. To investigate this, we first identified relapse of IgAN, defined as occurrence of proteinuria higher than $1 \mathrm{~g} / \mathrm{gCr}$ on two or more consecutive tests within 2 years after completion of immunosuppression. There were 2 (4.65\%), 1 (2.38\%), and $5(11.9 \%)$ relapses in Ter1, Ter2, and Ter3, respectively. There were fewer relapses in Terı than in Terз. Additionally, we conducted extended follow-up assessment of changes in TAP after year 1 (Supplementary Fig. 2). In Terı, TAP level decreased further at year 2 and then slightly increased thereafter. In contrast, there was a greater rebound increase in TAP in Ter2 from year 2, and Ter2 had a consistently high TAP level of $>1.0$ $\mathrm{g} / \mathrm{gCr}$. This reverse pattern in TAP level between Terı and Ter2 was maintained during the remaining observation period. In Ter3, TAP level did not decrease and increased further in year 6 .

\section{DISCUSSION}

In this study, we sought to evaluate long-term kidney outcomes according to the extent of proteinuria reduction after immunosuppressive therapy in patients with IgAN. First, we found that the rate of eGFR decline was significantly slower in patients with the greatest proteinuria reduction after immunosuppression. Second, when comparing eGFR by dividing the follow-up period into 1-year intervals, this slower eGFR decline in the group with greater proteinuria reduction persisted until 7 years after the start of immunosuppressive therapy. Last, the risk of disease progression was lower in 
Table 1. Baseline characteristics of patients with biopsy-proven IgA nephropathy who underwent immunosuppressive therapy

\begin{tabular}{|c|c|c|c|c|c|}
\hline \multirow[t]{2}{*}{ Characteristic } & \multirow{2}{*}{$\begin{array}{c}\text { Total } \\
(\mathrm{n}=127)\end{array}$} & \multicolumn{3}{|c|}{$\begin{array}{l}\text { Tertiles of proteinuria reduction after } \\
\text { immunosuppressive therapy }\end{array}$} & \multirow[t]{2}{*}{$p$ value } \\
\hline & & $\operatorname{Ter} 1(n=43)$ & $\operatorname{Ter} 2(\mathrm{n}=42)$ & $\operatorname{Ter} 3(n=42)$ & \\
\hline \multicolumn{6}{|l|}{ Demographic data } \\
\hline Age, yr & $41.2 \pm 11.4$ & $39.0 \pm 9.8$ & $40.7 \pm 11.0$ & $43.8 \pm 13.0$ & 0.150 \\
\hline Proteinuria, g/gCr & $2.1(1.4-3.5)$ & $3.7(2.6-5.2)$ & $1.5(1.3-2.0)$ & $2.0(1.2-2.8)$ & $<0.001$ \\
\hline Pre-IS TAP, g/gCr & $1.9(1.4-3.1)$ & $3.3(2.7-4.9)$ & $1.5(1.3-1.9)$ & $1.5(1.0-2.1)$ & $<0.001$ \\
\hline Post-IS TAP, g/gCr & $1.1(0.6-1.6)$ & $1.2(0.7-1.6)$ & $0.7(0.4-0.9)$ & $1.6(0.9-2.3)$ & $<0.001$ \\
\hline Reduction of proteinuria, g/gCr & $-0.9(-1.8$ to -0.4$)$ & $-2.1(-3.7$ to -1.5$)$ & $-0.9(-1.0$ to -0.7$)$ & $-0.2(-0.4$ to 0.5$)$ & \\
\hline $\mathrm{SBP}, \mathrm{mmHg}$ & $122.9 \pm 13.8$ & $122.4 \pm 16.9$ & $121.9 \pm 11.2$ & $124.4 \pm 12.5$ & 0.710 \\
\hline DBP, $\mathrm{mmHg}$ & $77.0 \pm 9.1$ & $76.0 \pm 9 \cdot 3$ & $76.9 \pm 8.4$ & $78.2 \pm 9.5$ & 0.560 \\
\hline \multicolumn{6}{|l|}{ Laboratory parameter } \\
\hline BUN, mg/dL & $16.8(13 \cdot 5-22.1)$ & $16.2(12.9-20.1)$ & $17.1(14.4-21.0)$ & $16.9(15.1-24.3)$ & 0.120 \\
\hline Creatinine, mg/dL & $1.1(0.8-1.4)$ & $1.0(0.7-1.3)$ & $1.0(0.8-1.5)$ & $1.3(0.9-1.6)$ & 0.009 \\
\hline $\mathrm{eGFR}, \mathrm{mL} / \mathrm{min} / 1.73 \mathrm{~m}^{2}$ & $70.2(51.6-100.3)$ & $80.6(64.7-102.1)$ & $73.2(52.5-93.1)$ & $59.1(43.6-85.2)$ & 0.018 \\
\hline Hemoglobin, g/dL & $12.6 \pm 1.8$ & $12.4 \pm 1.6$ & $12.9 \pm 1.8$ & $12.6 \pm 2.0$ & 0.410 \\
\hline Calcium, mg/dL & $8.9(8.6-9.1)$ & $8.7(8.5-9.0)$ & $8.9(8.8-9.2)$ & $8.9(8.7-9.1)$ & 0.023 \\
\hline Inorganic phosphorus, mg/dL & $3.7 \pm 0.5$ & $3.8 \pm 0.5$ & $3.6 \pm 0.4$ & $3.8 \pm 0.6$ & 0.400 \\
\hline Uric acid, mg/dL & $6.0(4.9-7.4)$ & $5.6(4.6-7.1)$ & $6.3(5.2-7.1)$ & $6.5(5 \cdot 5-7.8)$ & 0.080 \\
\hline Total protein, g/dL & $6.4(6.1-6.8)$ & $6.2(5.5-6.5)$ & $6.7(6.3-6.9)$ & $6.5(6.2-6.8)$ & 0.003 \\
\hline Albumin, g/dL & $3.8(3.4-4.0)$ & $3.5(3.2-3.8)$ & $4.0(3.7-4.1)$ & $3.8(3.4-4.0)$ & $<0.001$ \\
\hline Total cholesterol, mg/dL & $194.0(173.0-226.0)$ & $209.0(181.0-249.0)$ & $186.5(163.0-207.0)$ & $194.5(173.0-237.0)$ & 0.017 \\
\hline $\mathrm{HDL}-\mathrm{C}, \mathrm{mg} / \mathrm{dL}$ & $49.0(40.0-61.0)$ & $49.5(41.0-63.0)$ & $44.0(38.5-53.5)$ & $51.0(45.0-62.5)$ & 0.220 \\
\hline LDL-C, mg/dL & $111.0(91.0-127.0)$ & $118.0(83.0-164.0)$ & $108.5(93.0-127.0)$ & $111.0(91.0-118.0)$ & 0.600 \\
\hline Triglyceride, mg/dL & $123.0(91.0-201.0)$ & $161.0(96.0-220.0)$ & $123.0(90.0-193.0)$ & $112.0(76.0-172.0)$ & 0.140 \\
\hline $\mathrm{Na}, \mathrm{mmol} / \mathrm{L}$ & $140.7 \pm 1.8$ & $140.5 \pm 1.9$ & $140.8 \pm 1.7$ & $140.6 \pm 1.8$ & 0.710 \\
\hline $\mathrm{K}, \mathrm{mmol} / \mathrm{L}$ & $4.5 \pm 0.4$ & $4.5 \pm 0.3$ & $4.4 \pm 0.4$ & $4.6 \pm 0.5$ & 0.048 \\
\hline $\mathrm{Cl}, \mathrm{mmol} / \mathrm{L}$ & $104.4 \pm 2.4$ & $104.8 \pm 2.4$ & $104.0 \pm 2.5$ & $104.5 \pm 2.5$ & 0.400 \\
\hline $\mathrm{tCO}_{2}, \mathrm{mmol} / \mathrm{L}$ & $25.1 \pm 2.3$ & $24.6 \pm 2.5$ & $25.4 \pm 2.3$ & $25.2 \pm 2.2$ & 0.280 \\
\hline \multicolumn{6}{|l|}{ Medication history } \\
\hline ACE inhibitor & $4(3.1)$ & 0 & $2(4.8)$ & $2(4.8)$ & 0.350 \\
\hline $\mathrm{ARB}$ & $91(71.7)$ & $30(69.8)$ & $33(78.6)$ & $28(66.7)$ & 0.450 \\
\hline Spironolactone & $4(3.1)$ & $2(4 \cdot 7)$ & $2(4.8)$ & 0 & 0.540 \\
\hline Statin & $35(27.6)$ & $15(34 \cdot 9)$ & $11(26.2)$ & $9(21.4)$ & 0.370 \\
\hline \multicolumn{6}{|l|}{ Biopsy findings } \\
\hline $\mathrm{M} 1$ & $46(36.2)$ & $14(32.6)$ & $11(26.2)$ & $21(50.0)$ & 0.063 \\
\hline $\mathrm{E} 1$ & $50(39.4)$ & $17(39 \cdot 5)$ & $21(50.0)$ & $12(28.6)$ & 0.130 \\
\hline $\mathrm{S}_{1}$ & $109(85.8)$ & $39(90.7)$ & $33(78.6)$ & $37(88.1)$ & 0.240 \\
\hline $\mathrm{T} 1$ & $22(17 \cdot 3)$ & $7(16.3)$ & $9(21.4)$ & $6(14 \cdot 3)$ & 0.670 \\
\hline $\mathrm{C} 1$ & $34(26.8)$ & $8(18.6)$ & $16(38.1)$ & $10(23.8)$ & 0.280 \\
\hline $\mathrm{C}_{2}$ & $3(2.4)$ & $2(4.7)$ & o & $1(2.4)$ & \\
\hline
\end{tabular}


Table 1. Continued

\begin{tabular}{|c|c|c|c|c|c|}
\hline \multirow[t]{2}{*}{ Characteristic } & \multirow{2}{*}{$\begin{array}{c}\text { Total } \\
(\mathrm{n}=127)\end{array}$} & \multicolumn{3}{|c|}{$\begin{array}{l}\text { Tertiles of proteinuria reduction after } \\
\text { immunosuppressive therapy }\end{array}$} & \multirow[t]{2}{*}{$p$ value } \\
\hline & & Ter1 $(n=43)$ & $\operatorname{Ter} 2(n=42)$ & Ter3 $(n=42)$ & \\
\hline \multicolumn{6}{|l|}{ Immunosuppressants } \\
\hline \multicolumn{6}{|l|}{ Number of patients } \\
\hline Glucocorticoid & $127(100.0)$ & $43(100.0)$ & $42(100.0)$ & $42(100.0)$ & \\
\hline Cyclophosphamide & $2(1.6)$ & $1(2.3)$ & o & $1(2.4)$ & 1.000 \\
\hline Mycophenolate & $6(4 \cdot 7)$ & $2(4 \cdot 7)$ & $1(2.4)$ & $3(7 \cdot 1)$ & 0.700 \\
\hline Cyclosporine & $27(21.3)$ & $12(27.9)$ & $4(9 \cdot 5)$ & $11(26.2)$ & 0.074 \\
\hline \multicolumn{6}{|l|}{ Total cumulative dose, g } \\
\hline Glucocorticoid & $7.8(5.6-12.6)$ & $8.3\left(6.5^{-13.1)}\right.$ & $7 \cdot 5(5.0-12.6)$ & $7 \cdot 7(5 \cdot 3-10.6)$ & 0.420 \\
\hline Cyclophosphamide & $9.0(5 \cdot 5-12.6)$ & 12.60 & None & 5.45 & \\
\hline Mycophenolate & $95.6(87.0-149.4)$ & $140.8(81.5-200.0)$ & $92.5^{\mathrm{a}}$ & $98.8(97.8-99.8)$ & 0.860 \\
\hline Cyclosporine & $55.9(25.1-85.9)$ & $55.9(37.8-87.0)$ & $69.5(43.5-74.4)$ & $52.9(11.1-82.8)$ & 0.710 \\
\hline $\begin{array}{l}\text { Duration of } \\
\text { immunosuppression, mon }\end{array}$ & $9.0(6.6-16.0)$ & $9.7(7.7-17.6)$ & $8.9(6.5-10.4)$ & $9.6(6.2-20.2)$ & 0.280 \\
\hline $\begin{array}{l}\text { Time from biopsy to start of } \\
\text { immunosuppression, mon }\end{array}$ & $1.4(0.5-18.0)$ & $0.6(0.5-1.7)$ & $1.2(0.5-9.4)$ & $15.7(6.3-56.2)$ & $<0.001$ \\
\hline
\end{tabular}

Values are presented as mean \pm standard deviation, median (interquartile range), or number (\%).

IgA, immunoglobulin A; Ter1, first tertile; Ter2, second tertile; Ter3, third tertile; pre-IS TAP, time-averaged proteinuria before the start of immunosuppressive therapy; post-IS TAP, time-averaged proteinuria within 1 year from the start of immunosuppressive therapy; SBP, systolic blood pressure; DBP, diastolic blood pressure; BUN, blood urea nitrogen; eGFR, estimated glomerular filtration rate; HDL-C, high-density lipoprotein cholesterol; LDL-C, low-density lipoprotein cholesterol; ACE, angiotensin converting enzyme; ARB, angiotensin receptor blocker.

${ }^{a}$ Only one patient was treated with mycophenolate at a mean dose of $92.5 \mathrm{~g}$.

Table 2. Post-treatment slopes of eGFR and comparison of TAP before and after immunosuppressive therapy

\begin{tabular}{|c|c|c|c|c|c|}
\hline Variable & No. of cases & $\begin{array}{l}\text { Post-treatment slope } \\
\qquad(95 \% \mathrm{CI})^{\mathrm{a}}\end{array}$ & $\begin{array}{l}\text { TAP before } 1 \text { year from treat- } \\
\text { ment initiation (IQR) }\end{array}$ & $\begin{array}{l}\text { TAP within } 1 \text { year from treat- } \\
\text { ment initiation (IQR) }\end{array}$ & $p$ value $^{\mathrm{b}}$ \\
\hline Overall & 127 & $-2.97(-3.99$ to -1.95$)$ & $1.9(1.4-3.1)$ & $1.1(0.6-1.6)$ & $<0.001$ \\
\hline Ter1 & 43 & $-2.03(-2.95$ to -1.11$)$ & $3.3(2.7-4.9)$ & $1.2(0.7-1.6)$ & $<0.001$ \\
\hline Ter2 & 42 & $-2.44(-4.71$ to -0.17$)$ & $1.5(1.3-1.9)$ & $0.7(0.4-0.9)$ & $<0.001$ \\
\hline Ter3 & 42 & $-4.62(-6.52$ to -2.72$)$ & $1.5(1.0-2.1)$ & $1.6(0.9-2.3)$ & 0.975 \\
\hline
\end{tabular}

eGFR, estimated glomerular filtration rate; TAP, time-averaged proteinuria; CI, confidence interval; IQR, interquartile range; Ter1, first tertile; Ter2, second tertile; Ter3, third tertile.

${ }^{a}$ The slope of eGFR was derived 12 months after the start of immunosuppressive therapy. Adjusted for age at start of immunosuppression, sex, time from biopsy to start of immunosuppressive therapy, baseline eGFR, TAP before the start of immunosuppressive therapy, time-averaged systolic blood pressure, serum albumin, and presence of endocapillary hypercellularity. ${ }^{\mathrm{b}} \mathrm{A} p$ value was derived from a Wilcoxon signed rank test.

patients with the greatest proteinuria reduction. These findings suggest that proteinuria reduction after immunosuppression can be a surrogate marker for responsiveness to immunosuppression and a useful predictor of long-term outcomes in patients with IgAN.
Proteinuria is a sign of kidney damage and a wellknown indicator of severity and prognosis in various renal diseases [22-25]. In IgAN, proteinuria is the strongest prognostic factor for adverse kidney outcomes, and reducing proteinuria to less than $1.0 \mathrm{~g} /$ day has been sug- 
gested to delay disease progression [9,26,27]. Consistent with this, our findings highlight the importance of proteinuria reduction as a surrogate marker of responsiveness to immunosuppression. Several other indicators of steroid responsiveness have been proposed, but findings have been inconsistent. For example, an earlier study by the Working Group of the International IgA Nephropathy Network and the Renal Pathology Society showed that the presence of endocapillary hypercellularity im-

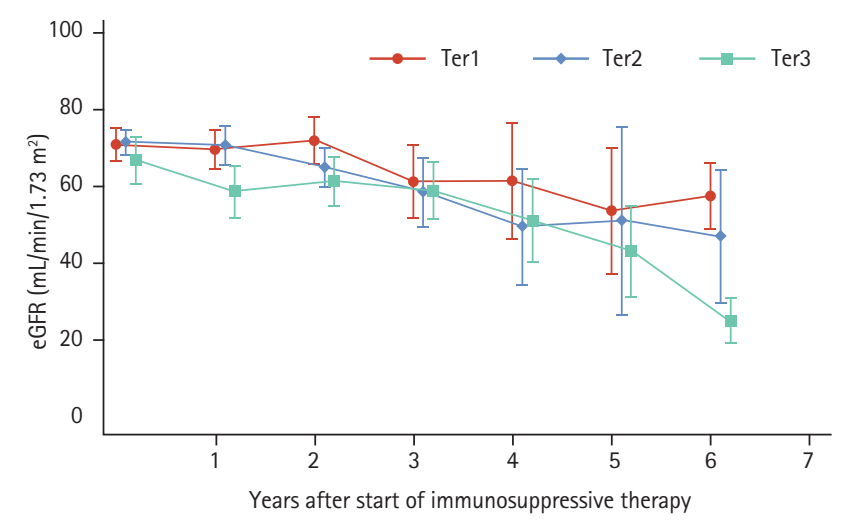

Figure 2. Changes in predicted estimated glomerular filtration rate (eGFR) using a piecewise linear mixed model. Patients were categorized into tertiles according to the extent of time-averaged proteinuria reduction after immunosuppression. The linear mixed model was used to derive the predicted eGFR after adjusting for age at start of treatment, sex, time from biopsy to start of immunosuppressive therapy, baseline eGFR, time-averaged proteinuria before the start of immunosuppressive therapy, time-averaged systolic blood pressure, serum albumin, and presence of endocapillary hypercellularity. Markers and capped spikes represent means and $95 \%$ confidence intervals. Ter1, first tertile; Ter2, second tertile; Ter3, third tertile. plies a good response to corticosteroids [13]. However, endocapillary hypercellularity has not been validated as a discriminator for treatment response by other cohort studies [28-30]. Nevertheless, from a practical viewpoint, repeated kidney biopsy is not widely performed and it is difficult to determine a treatment response from biopsy findings. In contrast, measurement of proteinuria is non-invasive and easily performed in clinical practice. Thus, serial monitoring of changes in proteinuria can be implemented to evaluate responsiveness to immunosuppression. We further showed that the extent of proteinuria reduction correlated well with the slopes of eGFR decline and was a good predictor of future adverse kidney outcomes, suggesting that proteinuria reduction is a useful marker of disease progression. Based on our results, we strongly support the recent suggestion by Thompson et al. [31], that proteinuria reduction is a reasonable endpoint to evaluate the effect of treatment in future clinical interventional trials.

It should be noted that patients in Terr showed slower disease progression than those in Ter2 despite the lower achieved proteinuria level in Ter2. To determine why this might be the case, we performed an extended follow-up assessment of changes in proteinuria after the completion of immunosuppression. Interestingly, proteinuria was further decreased in Terı at year 2, whereas there was a rebound increase in proteinuria in Ter2 at year 2. During the remaining observation period, patients in Ter2 had a higher proteinuria level (> 1.0 g/gCr) than patients in Terı. Not surprisingly, persistently elevated proteinuria $>1.0 \mathrm{~g} /$ day is a poor prognostic fac-

Table 3. Cox proportional hazard model of disease progression ${ }^{\mathrm{a}}$ according to reduction of proteinuria after immunosuppressive therapy

\begin{tabular}{|c|c|c|c|c|c|c|c|c|}
\hline \multirow{2}{*}{ Variable } & \multicolumn{2}{|c|}{ Model 1} & \multicolumn{2}{|c|}{ Model 2} & \multicolumn{2}{|l|}{ Model 3} & \multicolumn{2}{|c|}{ Model $_{4}$} \\
\hline & $\mathrm{HR}(95 \% \mathrm{CI})$ & $p$ value & $\mathrm{HR}(95 \% \mathrm{CI})$ & $p$ value & $\operatorname{HR}(95 \% \mathrm{CI})$ & $p$ value & $\mathrm{HR}(95 \% \mathrm{CI})$ & $p$ value \\
\hline Terı & $0.51(0.26-0.98)$ & 0.045 & $0.51(0.23-1.13)$ & 0.096 & $0.34(0.14-0.82)$ & 0.016 & $0.30(0.12-0.74)$ & 0.009 \\
\hline Ter2 & $0.70(0.36-1.35)$ & 0.285 & $0.66\left(0.33^{-1.33)}\right.$ & 0.248 & $0.78(0.38-1.61)$ & 0.504 & $0.70(0.34-1.45)$ & 0.339 \\
\hline Ter3 & Reference & - & Reference & - & Reference & - & Reference & - \\
\hline
\end{tabular}

Model 1: unadjusted; Model 2: adjusted for age at start of immunosuppression, sex, time from biopsy to start of immunosuppressive therapy, and baseline estimated glomerular filtration rate (eGFR); Model 3: Model 2 + time-averaged proteinuria before the start of immunosuppressive therapy and time-averaged systolic blood pressure; Model 4: Model $3+$ serum albumin, presence of endocapillary hypercellularity and use of renin-angiotensin system blocker at start of immunosuppressive therapy. HR, hazard ratio; CI, confidence interval; Ter1, first tertile; Ter2, second tertile; Ter3, third tertile.

${ }^{a}$ Disease progression was defined as the occurrence of $\geq 30 \%$ decline in eGFR or end-stage renal disease. 


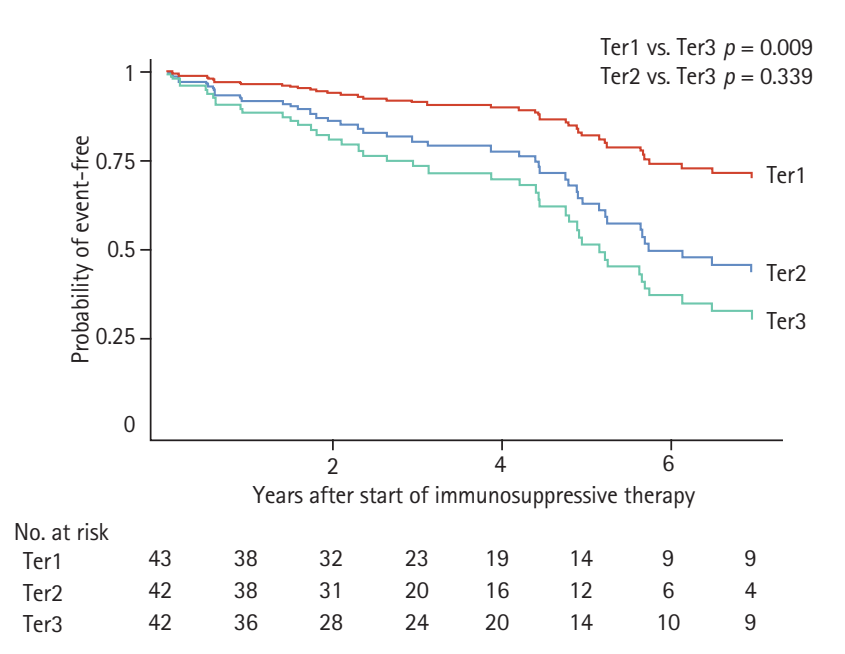

Figure 3. Adjusted survival curves of disease progression (disease progression was defined as the occurrence of a $30 \%$ or greater decline in estimated glomerular filtration rate [eGFR] or end-stage renal disease) for patients with immunoglobulin A nephropathy who underwent immunosuppressive therapy. Patients were categorized into tertiles according to the extent of time-averaged proteinuria reduction after immunosuppression. Adjustments were made for age at start of treatment, sex, time from biopsy to start of treatment, baseline eGFR, time-averaged proteinuria before the start of immunosuppressive therapy, time-averaged systolic blood pressure, serum albumin, presence of endocapillary hypercellularity and use of renin-angiotensin system blocker at start of immunosuppressive therapy. Terı, first tertile; Ter2, second tertile; Ter3, third tertile.

tor $[16,32]$. This finding can explain the higher risk of disease progression in the Ter2 group in our study, and underscores the importance of a sustained decrease in proteinuria in IgAN.

Another important finding to note is that patients with the greatest proteinuria reduction in response to immunosuppression had greater preservation of their eGFR at the start of immunosuppression and a shorter time from biopsy to commencement of immunosuppression. These findings have several important clinical implications. First, our results support the current guidelines suggesting that immunosuppression be considered in patients with preserved kidney function $\left(\right.$ eGFR $\geq 50 \mathrm{~mL} / \mathrm{min} / 1.73 \mathrm{~m}^{2}$ ) and be avoided in patients with advanced stages of CKD. Because fibrosis is considered an irreversible change and immunosuppression is not effective in patients with severe fibrosis, we excluded eight patients with eGFR $<30 \mathrm{~mL} / \mathrm{min} / 1.73 \mathrm{~m}^{2}$ and a T2 score from our analyses. Despite similar proportions

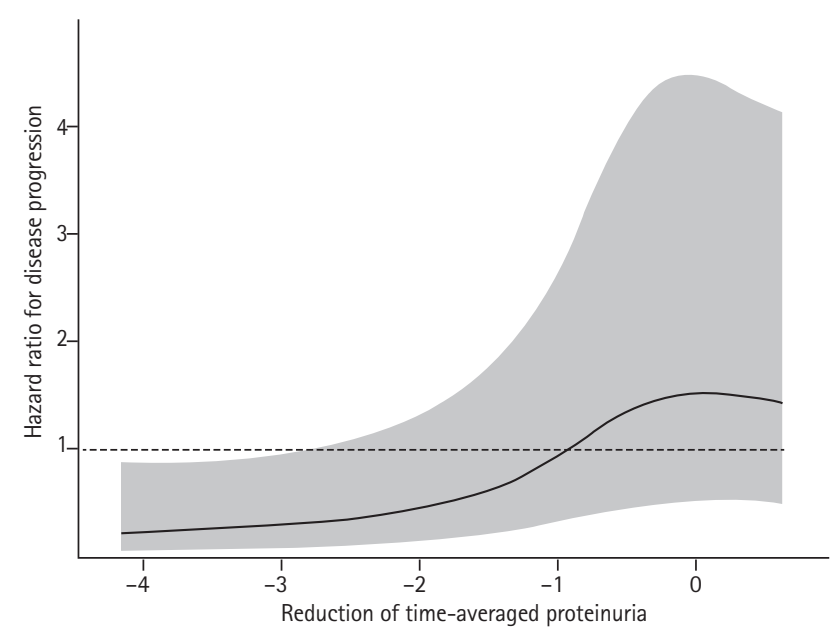

Figure 4. Restricted cubic spline curve for disease progression (disease progression was defined as the occurrence of a $30 \%$ or greater decline in estimated glomerular filtration rate [eGFR] or end-stage renal disease) according to the extent of time-averaged proteinuria reduction. The shaded area represents the $95 \%$ confidence interval. Adjustments were made for age at start of treatment, sex, time from biopsy to start of treatment, baseline eGFR, time-averaged proteinuria before the start of immunosuppressive therapy, time-averaged systolic blood pressure, serum albumin, presence of endocapillary hypercellularity and use of renin-angiotensin system blocker at start of immunosuppressive therapy.

of T1 scores and other pathologic lesions among tertile groups, we found that responsiveness to immunosuppression was greater in patients with a higher eGFR at baseline. Thus, treatment responses are likely to be better in patients with preserved kidney function. Second, based on our findings, early commencement of immunosuppression may be more effective in IgAN than late commencement. Few studies have examined the timing of initiation of immunosuppression. In general, IgAN is an indolent disease and rapid progression rarely occurs. Thus, it is particularly difficult to determine when to commence immunosuppression in patients with an eGFR of $60 \mathrm{~mL} / \mathrm{min} / 1.73 \mathrm{~m}^{2}$ and proteinuria of $1.0 \mathrm{~g} /$ day. Such patients would likely experience unfavorable outcomes if proteinuria reduction is not achieved given the preexisting evidence that risk of disease progression increases markedly in patients with proteinuria $\geq 1.0 \mathrm{~g} /$ day $[16,18]$. It is possible that glomerular inflammation and tubular injury may still be ongoing despite maximal 
supportive care such as the use of RAAS blockers and a low protein diet. Future trials should address whether early initiation of immunosuppression can improve kidney outcomes.

There have been attempts to use the Oxford-MESTC score for risk stratification in IgAN. However, no consensus has yet been reached regarding this issue. Only the T-score has been shown to consistently predict adverse kidney outcomes, and studies to date have yielded conflicting results on which pathologic components can convincingly predict disease progression [33]. As described above, we found no significant difference in Oxford-MESTC score among tertile groups according to the extent of proteinuria reduction. Studies on the relationship between the Oxford score and responsiveness to immunosuppression are scarce. The initial Working Group study showed a significant interaction between endocapillary hypercellularity and corticosteroid use, but this requires further validation [13]. Finding pathologic features that can correctly predict treatment response is very important, because utilization of such lesions can aid in decision-making with regards to the timing of initiation of immunosuppression. Unfortunately, no randomized controlled trials have been conducted to address this issue. In this regard, we are awaiting the results from the ongoing Treatment of IgA nEphropathy according to Renal lesions (TIGER) study, which is a prospective, open-labelled, randomized controlled study to evaluate the efficacy of early corticosteroid therapy combined with RAAS blockade (versus RAAS blockade alone) after 2 years of evolution in IgAN patients with severe histological lesions [34].

This study has several limitations. First, it was an observational study with a relatively small sample size. Thus, the influences of confounding factors could not be controlled for and causality between treatment and outcome could not be determined. Baseline characteristics did not differ much among tertile groups, but it is possible that other unmeasured factors might have affected proteinuria reduction. Second, the immunosuppression protocol was not uniform and there was no pre-set regimen, dosage, or duration of immunosuppression although all patients were initially treated with corticosteroids based on similar protocols suggested by the current KDIGO guidelines [9]. In addition, further immunosuppression with other drugs was allowed at the physician's discretion. Future studies should use a well-designed immunosuppression protocol to robustly determine the relationship between the use of immunosuppression and proteinuria reduction. Third, our database did not include detailed information on adverse side effects of immunosuppression. We reviewed medical records and found five events of severe infection and seven events of new diabetes mellitus during the follow-up period. The incidence rate of severe infection in our study was lower than that reported in previous randomized controlled studies [10,11], possibly because we routinely administered prophylactic antibiotics to all patients during immunosuppressive treatment period. Nevertheless, fatal adverse effects of immunosuppression should not outweigh the effect of immunosuppression on delaying disease progression. Finally, our study was not designed to compare treatment effects between immunosuppression and supportive care. However, proteinuria reduction can be achieved spontaneously or with intensive supportive care. Further studies are needed to evaluate the relationship between proteinuria reduction and risk of long-term adverse kidney outcomes in IgAN patients.

In conclusion, we showed that greater proteinuria reduction after immunosuppression was associated with a lower risk of disease progression in IgAN, suggesting that proteinuria reduction after immunosuppression may be a surrogate marker for responsiveness to immunosuppression and a useful predictor of long-term kidney outcomes.

\section{KEY MESSAGE}

1. Greater proteinuria reduction after immunosuppressive therapy is associated with a lower risk of disease progression in immunoglobulin A nephropathy.

2. Proteinuria reduction after immunosuppressive therapy may be a surrogate marker for responsiveness to immunosuppression and predictor of long-term kidney outcomes.

\section{Conflict of interest}

No potential conflict of interest relevant to this article was reported. 


\section{Acknowledgments}

This research was supported by the research fund of the Korea Centers for Disease Control and Prevention (2019ER690101).

\section{REFERENCES}

1. Schena FP, Nistor I. Epidemiology of IgA nephropathy: a global perspective. Semin Nephrol 2018;38:435-442.

2. D'Amico G. The commonest glomerulonephritis in the world: IgA nephropathy. Q J Med 1987;64:709-727.

3. Schena FP. A retrospective analysis of the natural history of primary IgA nephropathy worldwide. Am J Med 1990;89:209-215.

4. Donadio JV, Grande JP. IgA nephropathy. N Engl J Med 2002;347:738-748.

5. Suzuki H, Fan R, Zhang Z, et al. Aberrantly glycosylated IgAn in IgA nephropathy patients is recognized by IgG antibodies with restricted heterogeneity. J Clin Invest 2009;119:1668-1677.

6. Pozzi C, Bolasco PG, Fogazzi GB, et al. Corticosteroids in IgA nephropathy: a randomised controlled trial. Lancet 1999;353:883-887.

7. Manno C, Torres DD, Rossini M, Pesce F, Schena FP. Randomized controlled clinical trial of corticosteroids plus ACE-inhibitors with long-term follow-up in proteinuric IgA nephropathy. Nephrol Dial Transplant 2009;24:36943701.

8. Lv J, Zhang H, Chen Y, et al. Combination therapy of prednisone and ACE inhibitor versus ACE-inhibitor therapy alone in patients with IgA nephropathy: a randomized controlled trial. Am J Kidney Dis 2009;53:26-32.

9. Kidney disease: Improving global outcomes (KDIGO) glomerulonephritis work group. KDIGO clinical practice guideline for glomerulonephritis. Kidney Int Suppl 2012;2:139-274.

10. Lv J, Zhang H, Wong MG, et al. Effect of oral methylprednisolone on clinical outcomes in patients with IgA nephropathy: the testing randomized clinical trial. JAMA 2017;318:432-442.

11. Rauen T, Eitner F, Fitzner C, et al. Intensive supportive care plus immunosuppression in IgA nephropathy. N Engl J Med 2015;373:2225-2236.

12. Floege J, Barbour SJ, Cattran DC, et al. Management and treatment of glomerular diseases (part 1): conclu- sions from a Kidney Disease: Improving Global Outcomes (KDIGO) Controversies Conference. Kidney Int 2019;95:268-280.

13. Working Group of the International IgA Nephropathy Network and the Renal Pathology Society, Cattran DC, Coppo R, et al. The Oxford classification of IgA nephropathy: rationale, clinicopathological correlations, and classification. Kidney Int 2009;76:534-545.

14. Chen T, Xia E, Chen T, et al. Identification and external validation of IgA nephropathy patients benefiting from immunosuppression therapy. EBioMedicine 2020;52:102657.

15. Le W, Liang S, Hu Y, et al. Long-term renal survival and related risk factors in patients with IgA nephropathy: results from a cohort of 1155 cases in a Chinese adult population. Nephrol Dial Transplant 2012;27:1479-1485.

16. Reich HN, Troyanov S, Scholey JW, Cattran DC; Toronto Glomerulonephritis Registry. Remission of proteinuria improves prognosis in IgA nephropathy. J Am Soc Nephrol 2007;18:3177-3183.

17. Mackinnon B, Fraser EP, Cattran DC, Fox JG, Geddes CC. Validation of the Toronto formula to predict progression in IgA nephropathy. Nephron Clin Pract 2008;109:c148-c153.

18. Nam KH, Kie JH, Lee MJ, et al. Optimal proteinuria target for renoprotection in patients with IgA nephropathy. PLoS One 2014;9:e101935.

19. Levey AS, Stevens LA, Schmid CH, et al. A new equation to estimate glomerular filtration rate. Ann Intern Med 2009;150:604-612.

20. Working Group of the International IgA Nephropathy Network and the Renal Pathology Society, Roberts IS, Cook HT, et al. The Oxford classification of IgA nephropathy: pathology definitions, correlations, and reproducibility. Kidney Int 2009;76:546-556.

21. Levey AS, Gansevoort RT, Coresh J, et al. Change in albuminuria and GFR as end points for clinical trials in early stages of CKD: a scientific workshop sponsored by the National Kidney Foundation in collaboration with the US Food and Drug Administration and European Medicines Agency. Am J Kidney Dis 2020;75:84-104.

22. D’Amico G, Bazzi C. Pathophysiology of proteinuria. Kidney Int 2003;63:809-825.

23. Gorriz JL, Martinez-Castelao A. Proteinuria: detection and role in native renal disease progression. Transplant Rev (Orlando) 2012;26:3-13. 
24. Nagata M. Podocyte injury and its consequences. Kidney Int 2016;89:1221-1230.

25. Kannel WB, Stampfer MJ, Castelli WP, Verter J. The prognostic significance of proteinuria: the Framingham study. Am Heart J 1984;108:1347-1352.

26. Kobayashi Y, Tateno S, Hiki Y, Shigematsu H. IgA nephropathy: prognostic significance of proteinuria and histological alterations. Nephron 1983;34:146-153.

27. Barbour SJ, Reich HN. Risk stratification of patients with IgA nephropathy. Am J Kidney Dis 2012;59:865-873.

28. Shi SF, Wang SX, Jiang L, et al. Pathologic predictors of renal outcome and therapeutic efficacy in IgA nephropathy: validation of the oxford classification. Clin J Am Soc Nephrol 2011;6:2175-2184.

29. Tesar V, Troyanov S, Bellur S, et al. Corticosteroids in IgA nephropathy: a retrospective analysis from the VALIGA Study. J Am Soc Nephrol 2015;26:2248-2258.

30. Shen XH, Liang SS, Chen HM, et al. Reversal of active glomerular lesions after immunosuppressive therapy in patients with IgA nephropathy: a repeat-biopsy based observation. J Nephrol 2015;28:441-449.

31. Thompson A, Carroll K, Inker LA, et al. Proteinuria reduction as a surrogate end point in trials of IgA nephropathy. Clin J Am Soc Nephrol 2019;14:469-481.

32. Berthoux F, Mohey H, Laurent B, Mariat C, Afiani A, Thibaudin L. Predicting the risk for dialysis or death in IgA nephropathy. J Am Soc Nephrol 2011;22:752-761.

33. Trimarchi H, Barratt J, Cattran DC, et al. Oxford classification of IgA nephropathy 2016: an update from the IgA nephropathy classification working group. Kidney Int 2017;91:1014-1021.

34. ClinicalTrials. Treatment of IgA Nephropathy According to Renal Lesions (TIGER) study [Internet]. Bethesda (MD): NIH, 2019 [cited 2020 Dec 29]. Available from: https:/clinicaltrials.gov/ct2/show/NCTo3188887?term=TIGER\&cond=IgA+Nephropathy\&draw=2\&rank=1. 
Supplementary Table 1. Baseline characteristics of patients with biopsy-proven immunoglobulin A nephropathy who underwent immunosuppressive therapy (at the time of kidney biopsy)

\begin{tabular}{|c|c|c|c|c|c|}
\hline \multirow[t]{2}{*}{ Variable } & \multirow{2}{*}{$\begin{array}{c}\text { Total } \\
(\mathrm{n}=127)\end{array}$} & \multicolumn{3}{|c|}{$\begin{array}{l}\text { Tertiles of proteinuria reduction after } \\
\text { immunosuppressive therapy }\end{array}$} & \multirow[t]{2}{*}{$p$ value } \\
\hline & & $\operatorname{Ter1}(n=43)$ & $\operatorname{Ter} 2(n=42)$ & $\operatorname{Ter} 3(n=42)$ & \\
\hline \multicolumn{6}{|l|}{ Demographic data } \\
\hline Proteinuria, g/gCr & $1.8(1.2-3.3)$ & $3.8(2.0-5.1)$ & $1.5(1.2-1.9)$ & $1.6(0.8-2.3)$ & $<0.001$ \\
\hline Age at biopsy, yr & $39.6 \pm 11.3$ & $38.3 \pm 9.9$ & $39.7 \pm 10.9$ & $41.0 \pm 12.9$ & 0.54 \\
\hline Female sex & $79(62.2)$ & $34(79.1)$ & $22(52.4)$ & $23(54.8)$ & 0.019 \\
\hline $\mathrm{BMI}, \mathrm{kg} / \mathrm{m}^{2}$ & $23.4(21.3-26.4)$ & $23.3(21.1-26.9)$ & $24.0(22.4-26.9)$ & $23.1(20.6-25.0)$ & 0.16 \\
\hline $\mathrm{SBP}, \mathrm{mmHg}$ & $121.2 \pm 14.6$ & $123.9 \pm 20.5$ & $120.9 \pm 11.3$ & $118.9 \pm 9.4$ & 0.34 \\
\hline $\mathrm{DBP}, \mathrm{mmHg}$ & $76.4 \pm 9.9$ & $78.0 \pm 11.0$ & $77 \cdot 3 \pm 9.2$ & $74.1 \pm 9.2$ & 0.19 \\
\hline \multicolumn{6}{|l|}{ Laboratory parameters } \\
\hline BUN, mg/dL & $15.7(13.0-18.5)$ & $13.9(12.4-17.3)$ & $16.5(13.0-19.8)$ & $16.7(14.9-19.1)$ & 0.043 \\
\hline Creatinine, mg/dL & $1.0(0.8-1.3)$ & $0.9(0.6-1.2)$ & $1.1(0.8-1.4)$ & $1.1(0.9-1.3)$ & 0.029 \\
\hline $\mathrm{eGFR}, \mathrm{mL} / \mathrm{min} / 1.73 \mathrm{~m}^{2}$ & $79 \cdot 3(61.2-103 \cdot 3)$ & $85.2(66.3-109.7)$ & $73.3(58.2-99 \cdot 3)$ & $76.0(64.4-93.8)$ & 0.18 \\
\hline Hemoglobin, g/dL & $12.9 \pm 1.8$ & $12.9 \pm 1.6$ & $13.2 \pm 1.9$ & $12.6 \pm 1.8$ & 0.28 \\
\hline Calcium, mg/dL & $8.9(8.6-9.3)$ & $8.8(8.5-9.1)$ & $9.1(8.6-9.3)$ & $9.0(8.7-9.3)$ & 0.052 \\
\hline Inorganic phosphorus, mg/dL & $3.7 \pm 0.6$ & $3.7 \pm 0.5$ & $3.6 \pm 0.6$ & $3.8 \pm 0.6$ & 0.31 \\
\hline Uric acid, mg/dL & $5.9(4.7-6.9)$ & $5 \cdot 3(4 \cdot 4-6.7)$ & $6.1(5.1-7.1)$ & $6.4(5.0-7.0)$ & 0.098 \\
\hline Total protein, g/dL & $6.4(6.1-6.9)$ & $6.3(5 \cdot 7-6.8)$ & $6.6(6.2-7.0)$ & $6.5(6.1-7.0)$ & 0.084 \\
\hline Albumin, g/dL & $3.8(3.5-4.1)$ & $3.6(3.2-4.0)$ & $3 \cdot 9(3.7-4.2)$ & $3.8(3.6-4.2)$ & 0.002 \\
\hline Total cholesterol, mg/dL & $201.0(174.0-235.0)$ & $221.0(201.0-270.0)$ & $192.5(178.0-214.0)$ & $186.0(164.0-223.0)$ & $<0.001$ \\
\hline $\mathrm{HDL}-\mathrm{C}, \mathrm{mg} / \mathrm{dL}$ & $48.5(40.0-61.0)$ & $51.5(41.5-61.5)$ & $41.0(40.0-57.0)$ & $53.0(41.0-64.0)$ & 0.38 \\
\hline $\mathrm{LDL}-\mathrm{C}, \mathrm{mg} / \mathrm{dL}$ & $121.0(106.0-150.0)$ & $126.0(116.0-181.0)$ & $110.5(93.0-142.0)$ & $125.0(110.0-135.0)$ & 0.11 \\
\hline Triglyceride, mg/dL & $140.0(93.0-219.0)$ & $148.5(108.0-220.0)$ & $186.0(115.0-244.0)$ & $118.0(76.0-148.0)$ & 0.053 \\
\hline $\mathrm{Na}, \mathrm{mmol} / \mathrm{L}$ & $140.7 \pm 1.9$ & $140.1 \pm 2.1$ & $141.0 \pm 2.0$ & $140.8 \pm 1.6$ & 0.088 \\
\hline $\mathrm{K}, \mathrm{mmol} / \mathrm{L}$ & $4.3 \pm 0.4$ & $4.3 \pm 0.4$ & $4.3 \pm 0.4$ & $4.3 \pm 0.4$ & 0.57 \\
\hline $\mathrm{Cl}, \mathrm{mmol} / \mathrm{L}$ & $104 \cdot 3 \pm 2 \cdot 3$ & $104.4 \pm 2.3$ & $104.1 \pm 2.2$ & $104.5 \pm 2.2$ & 0.77 \\
\hline $\mathrm{tCO} 2, \mathrm{mmol} / \mathrm{L}$ & $25.7 \pm 2.3$ & $25.6 \pm 2.3$ & $25.8 \pm 2.3$ & $25.7 \pm 2.4$ & 0.95 \\
\hline \multicolumn{6}{|l|}{ Comorbidities } \\
\hline $\mathrm{HTN}$ & $104(81.9)$ & $35(81.4)$ & $33(78.6)$ & $36(85.7)$ & 0.69 \\
\hline $\mathrm{DM}$ & $14(11.0)$ & $3(7.0)$ & $5(11.9)$ & $6(14 \cdot 3)$ & 0.53 \\
\hline CVD & $4(3.1)$ & o & $2(4.8)$ & $2(4.8)$ & 0.39 \\
\hline CHD & $4(3.1)$ & $2(4 \cdot 7)$ & 0 & $2(4.8)$ & 0.54 \\
\hline \multicolumn{6}{|l|}{ Medication history } \\
\hline ACE inhibitor & $5(3 \cdot 9)$ & o & $2(4.8)$ & $3(7.1)$ & 0.23 \\
\hline $\mathrm{ARB}$ & $78(61.4)$ & $20(46.5)$ & $29(69.0)$ & $29(69.0)$ & 0.047 \\
\hline Spironolactone & $6(4.7)$ & $3(7.0)$ & $1(2.4)$ & $2(4.8)$ & 0.87 \\
\hline Statin & $30(23.6)$ & $11(25.6)$ & $7(16.7)$ & $12(28.6)$ & 0.41 \\
\hline
\end{tabular}

Values are presented as median (interquartile range), mean \pm standard deviation, or number (\%).

Terı, first tertile; Ter2, second tertile; Ter3, third tertile; BMI, body mass index; SBP, systolic blood pressure; DBP, diastolic blood pressure; BUN, blood urea nitrogen; eGFR, estimated glomerular filtration rate; HDL-C, high-density lipoprotein cholesterol; LDL-C, low-density lipoprotein cholesterol; HTN, hypertension; DM, diabetes mellitus; CVD, cerebrovascular disease; CHD, coronary heart disease; ACE, angiotensin converting enzyme; ARB, angiotensin receptor blocker. 
Supplementary Table 2. Cox proportional hazard model of disease progressiona according to reduction of proteinuria after immunosuppressive therapy in subgroups divided by baseline proteinuria

\begin{tabular}{|c|c|c|c|c|c|c|}
\hline \multirow{2}{*}{ Variable } & \multicolumn{2}{|l|}{ Model 1} & \multicolumn{2}{|l|}{ Model 2} & \multicolumn{2}{|c|}{ Model $_{3}$} \\
\hline & $\operatorname{HR}(95 \% \mathrm{CI})$ & $p$ value & $\operatorname{HR}(95 \% \mathrm{CI})$ & $p$ value & $\operatorname{HR}(95 \% \mathrm{CI})$ & $p$ value \\
\hline \multicolumn{7}{|c|}{ Patients whose Pre-IS TAP $\geq 3.0 \mathrm{~g} / \mathrm{gCr}(\mathrm{n}=34)$} \\
\hline Ter1 & $0.88(0.25-3.08)$ & 0.846 & $0.83(0.16-4.14)$ & 0.816 & $0.54(0.09-3.05)$ & 0.484 \\
\hline Ter2 & $0.51(0.11-2.46)$ & 0.403 & $0.51(0.11-2.45)$ & 0.398 & $1.32(0.16-11.18)$ & 0.796 \\
\hline Ter3 & Reference & & Reference & & Reference & \\
\hline \multicolumn{7}{|c|}{ Patients whose Pre-IS TAP <3.0 g/gCr $(\mathrm{n}=93)$} \\
\hline Ter1 & $0.40(0.12-1.29)$ & 0.125 & $0.32(0.09-1.14)$ & 0.079 & $0.31(0.08-1.16)$ & 0.082 \\
\hline Ter2 & $0.90(0.43-1.87)$ & 0.769 & $0.98(0.47-2.08)$ & 0.968 & $0.98(0.47-2.05)$ & 0.951 \\
\hline Ter3 & Reference & & Reference & & Reference & \\
\hline
\end{tabular}

Model 1: adjusted for age at start of immunosuppression, sex, time from biopsy to start of immunosuppressive therapy, and baseline estimated glomerular filtration rate (eGFR); Model 2: Model 2 + pre-IS TAP and time-averaged systolic blood pressure; Model 3: Model $3+$ serum albumin, presence of endocapillary hypercellularity and use of renin-angiotensin system blocker at start of immunosuppressive therapy.

$\mathrm{HR}$, hazard ratio; CI, confidence interval; pre-IS TAP, time-averaged proteinuria before the start of immunosuppressive therapy; Terı, first tertile; Ter2, second tertile; Ter3, third tertile.

${ }^{a}$ Disease progression was defined as the occurrence of $\geq 30 \%$ decline in eGFR or end-stage renal disease. 

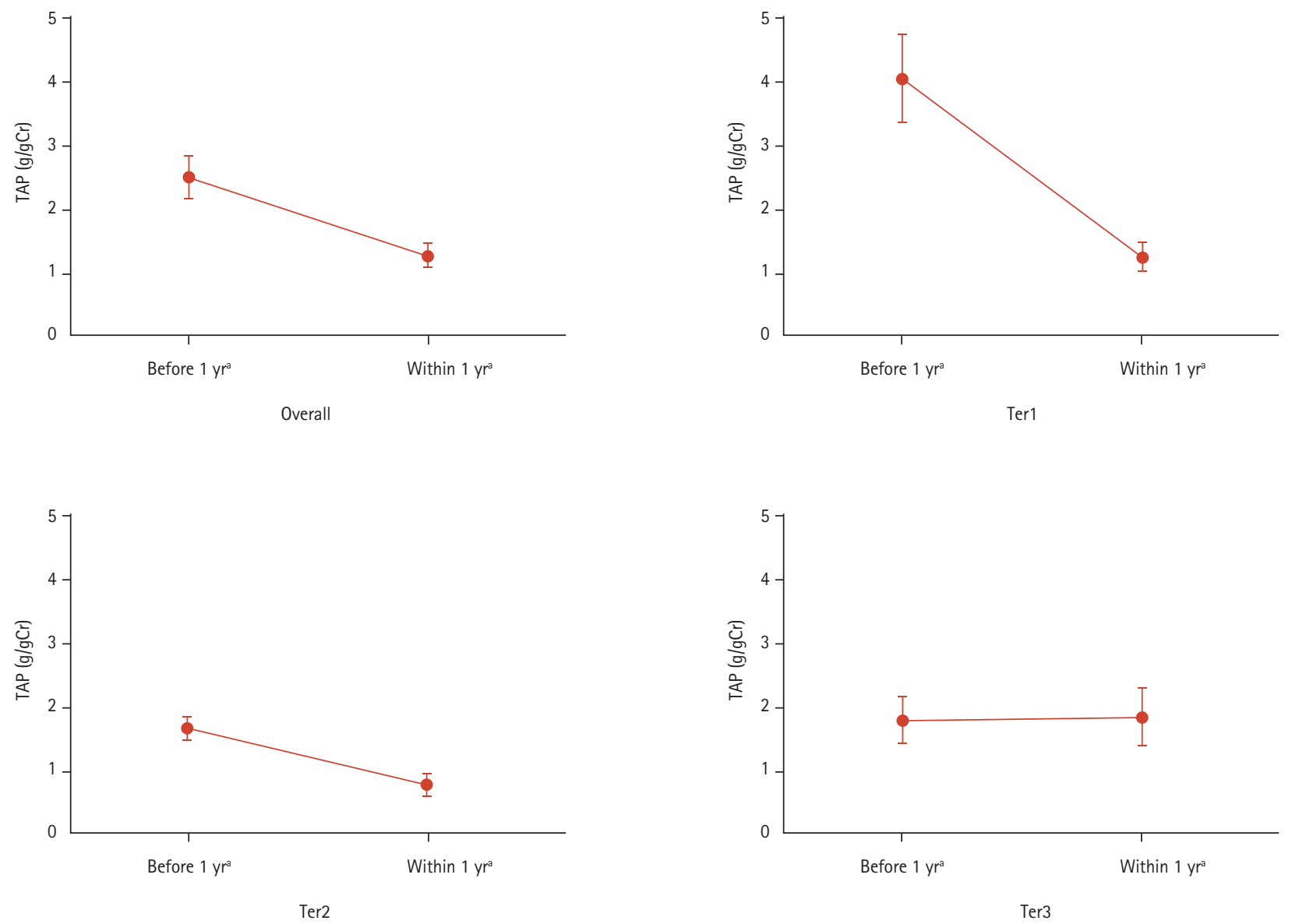

Supplementary Figure 1. Differences in time-averaged proteinuria within 12 months before and after the start of immunosuppression. Patients were categorized into tertiles according to the extent of time-averaged proteinuria reduction after immunosuppression. Markers and capped spikes represent means and 95\% confidence intervals. TAP, time-averaged proteinuria; Terı, first tertile; Ter2, second tertile; Ter3, third tertile. ${ }^{a}$ Time points were from the start of immunosuppression therapy. 


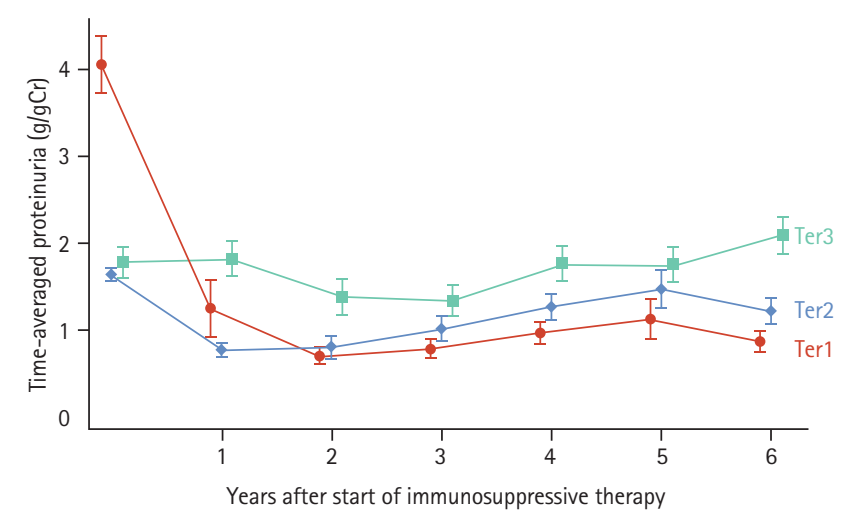

Supplementary Figure 2. Changes in time-averaged proteinuria in immunoglobulin A nephropathy patients who underwent immunosuppressive therapy. Patients were categorized into tertiles according to the extent of time-averaged proteinuria reduction after immunosuppression. Markers and capped spikes represents means and standard errors. Ter1, first tertile; Ter2, second tertile; Ter3, third tertile. 